\title{
In Situ Electrochemical AFM Imaging of a Pt Electrode in Sulfuric Acid under Potential Cycling Conditions
}

\author{
Xin Deng, ${ }^{\dagger}$ Federica Galli, ${ }^{\ddagger}$ and Marc T. M. Koper* ${ }^{*} \dagger$ \\ ${ }^{\dagger}$ Leiden Institute of Chemistry, Leiden University, PO Box 9502, 2300 RA, Leiden, The Netherlands \\ ${ }^{*}$ Huygens-Kamerlingh Onnes Laboratory, Leiden University, Niels Bohrweg 2, 2333 CA Leiden, The Netherlands
}

\section{Supporting Information}

ABSTRACT: Understanding the electrochemical behavior of $\mathrm{Pt}$ at the solid/liquid interface is of significant importance for the development of efficient electrochemical devices, such as fuel cells and water electrolyzers. In this work, the evolution of the surface morphology of a polycrystalline platinum under potential cycling conditions was investigated by in situ electrochemical atomic force microscopy (EC-AFM). After

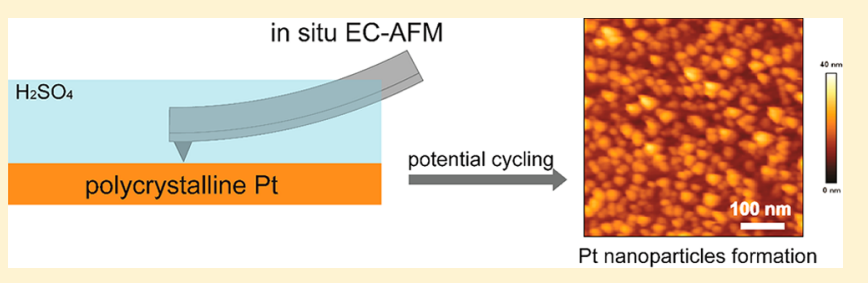
50 cycles between 0.05 and $1.8 \mathrm{~V}$ in $0.1 \mathrm{M} \mathrm{H}_{2} \mathrm{SO}_{4}$, the $\mathrm{Pt}$ surface is coarsened and nanoparticles of several nanometers appear on the surface. The critical upper and lower potentials for the formation of nanoparticles are found to be 1.8 and $0.8 \mathrm{~V}$, respectively. The in situ AFM observation coupled with Cyclic Voltammerty reveals the periodic disappearance and reappearance of the nanoparticles, based on which the formation of nanoparticles is attributed to the deposition of dissolved Pt from solution, and a model for the nanoparticle formation is proposed. While the formation of a thick oxide layer is a prerequisite, the reduction process is found to have a strong influence on $\mathrm{Pt}$ nanoparticle formation as well. This investigation provides a visualization of the Pt electrode surface under electrochemical control in a large potential window, enabling a broader understanding of the Pt electrode roughening mechanisms.

\section{INTRODUCTION}

An atomic level understanding of the surface oxidation and subsequent reduction processes at platinum electrodes is vital since $\mathrm{Pt}$ is one of the most widely used metals in the field of applied electrochemistry. Platinum is used as a cathode and anode material in acid fuel cells and water electrolyzers due to its excellent performance as a catalyst, as well as in many electroanalytical devices. However, the electrochemical stability of $\mathrm{Pt}$ is compromised by the formation of platinum oxides and their reduction, causing serious problems for practical operation. ${ }^{1,2}$ Platinum oxidation and subsequent reduction happen during stop-start cycles in practical devices and are typically simulated by continuous potential cycling in laboratory experiments. Much effort has been spent to prevent or diminish the degradation of $\mathrm{Pt}$ by enhancing the $\mathrm{Pt}-$ carbon interaction or by alloying Pt with other metals. ${ }^{3,4}$ Simultaneously, it is of significant importance to explore the underlying fundamental mechanisms for the electrochemical processes at $\mathrm{Pt}$ electrodes during potential cycling. The onset of the oxidation of polycrystalline $\mathrm{Pt}$ is at around $0.8 \mathrm{~V}$. The formation of a $2 \mathrm{D}$ platinum peroxide phase on $\mathrm{Pt}(111)$ single crystals was identified in the $1.0-1.1 \mathrm{~V}$ potential region by in situ Raman spectroscopy. ${ }^{5}$ During further surface oxidation, a $3 \mathrm{D} \alpha-\mathrm{PtO}_{2}$ oxide forms at $1.15 \mathrm{~V}$ via the so-called placeexchange mechanism, in which surface $\mathrm{Pt}$ atoms change their position with the oxygen derived from the oxidation of water. After reduction, some of these $\mathrm{Pt}$ atoms do not return to their original sites, rendering the process structurally irreversible and causing the roughening of the $\mathrm{Pt}$ surface. Dissolution of $\mathrm{Pt}$ electrodes during the surface roughening is an important cause for electrode instability. Pt dissolution in perchloric acid was investigated by online inductively coupled plasma mass spectrometry (ICP-MS), and it was found that the concentration of $\mathrm{Pt}$ ions in the solution depends on the dissolution potential and on the orientation of the $\mathrm{Pt}$ electrode. ${ }^{6}$ By utilizing an electrochemical scanning flow cell coupled to ICP-MS, Topalov et al. showed that under cycling conditions the dissolution mainly occurs in the cathodic sweep, i.e., during the reduction of the surface oxide, with the amount of dissolved Pt increasing with the upper potential limit. ${ }^{7}$

In situ observation of electrochemically induced roughening of $\mathrm{Pt}$ electrodes has been studied by scanning probe microscopy, either using scanning tunneling microscopy (STM) or atomic force microscopy (AFM). The surface roughening phenomenon of $\mathrm{Pt}$ single crystals after multiple oxidation-reduction cycles has been observed by electrochemical (EC)-STM. ${ }^{8-11}$ More recently, in situ EC-STM investigation of $\mathrm{Pt}(111)$ during oxidation-reduction cycles revealed the formation of $\mathrm{Pt}$ monatomic islands. ${ }^{12}$ After prolonged oxidation-reduction cycles, the islands contribute equally to the electrochemical signal in the hydrogen region and to the root-mean-square roughness derived from the STM images. ${ }^{13}$ From in situ grazing incidence small-angle X-ray

Received: July 15, 2018

Published: September 17, 2018 
scattering (GISAXS) measurements of a $\mathrm{Pt}(111)$ electrode in perchloric acid, it was concluded that the formation of $\mathrm{Pt}$ islands during oxidation-reduction cycles resembles the 3D growth behavior of $\mathrm{Pt}(111)$ during deposition and erosion in ultrahigh vacuum. ${ }^{11}$ Due to the requirement of conductivity, EC-STM experiments are normally performed with an upper limit of $1.3 \mathrm{~V}$. The utilization of EC-AFM enables the visualization of the dynamic process at the Pt surface in a wider potential range, and additionally, EC-AFM can be used to study processes on the surface that might not be ideal for STM, such as the imaging of a $\mathrm{Pt}$ film on carbon ${ }^{14}$ or $\mathrm{Pt}$ nanoparticles. ${ }^{15}$ However, there are only a few reports about the investigation of $\mathrm{Pt}$ electrodes using EC-AFM.

Here, we present an in situ EC-AFM study of the surface changes observed on a flame-annealed polycrystalline $\mathrm{Pt}$ electrode in sulfuric acid electrolyte subjected to repeated oxidation-reduction cycles. The most significant observation is the formation of $\mathrm{Pt}$ nanoparticles on the surface after multiple sweeps to sufficiently high potential. We show that the observation of these nanoparticles depends on the creation of Pt ions in the solution, which act as the source of nanoparticle generation during the reductive sweep, provided the sweep goes negative enough to reduce the surface oxide and provided that the Pt ions are not given the opportunity to diffuse away. This mechanism of surface roughening through (extensive) redeposition of dissolved platinum appears to be specific to polarization at higher anodic potential, a regime that was not included in previous studies of $\mathrm{Pt}$ coarsening studied by GISAXS, ${ }^{11}$ EC-STM, ${ }^{13}$ or EC-AFM. ${ }^{15}$ Our results illustrate the ability of in situ EC-AFM as a tool to investigate the coarsening behavior of the Pt electrode under electrochemical conditions and provide new insights into the phenomena taking place on the Pt electrode during oxidation-reduction cycles.

\section{EXPERIMENTAL SECTION}

The in situ AFM experiments were carried out in an electrochemical AFM cell that is made of PEEK. Before assembly, the cell components were cleaned by sonication in ethanol and Millipore Milli- $Q$ water (resistivity $18.2 \mathrm{M} \Omega \mathrm{cm}$ ), respectively, and then blow dried in air. Prior to each experiment, the working electrode Pt foil (99.9\%, MaTeck) was flame-annealed and quenched with Milli-Q water before assembling into the EC-AFM cell. A Ag/ $\mathrm{AgCl}(3 \mathrm{M} \mathrm{KCl})$ electrode (WPI) was used as the reference electrode, and the counter electrode was a Pt wire. All the potentials in this article are reported with respect to a reversible hydrogen electrode (RHE). A potentiostat ( $\mu$ Autolab type III) was coupled with the AFM (JPK NanoWizard 3) to control the electrochemical conditions during the experiments. The sweep rate was set to $100 \mathrm{mV} / \mathrm{s}$ for all the cyclic voltammetry measurements. The AFM scan rate was $1 \mathrm{~Hz}$, and all the images were obtained using tapping mode, to minimize the damage to the electrode. The tips used were purchased from Bruker (SNL, resonance frequency: $65 \mathrm{kHz}$, spring constant: $0.35 \mathrm{~N} / \mathrm{m}$ ). Images were taken either at $0.5 \mathrm{~V}$ after potential cycling or during cycling simultaneously with cyclic voltammetry (CV). The electrolyte was prepared from $\mathrm{H}_{2} \mathrm{SO}_{4}$ (Merk Ultrapur, 96\%), which was neither completely degassed nor refreshed during the experiment.

\section{RESULTS AND DISCUSSION}

Cyclic Voltammetry of the Flame-Annealed Pt in an EC-AFM Cell. The blank cyclic voltammogram of the flameannealed Pt electrode in $0.1 \mathrm{M} \mathrm{H}_{2} \mathrm{SO}_{4}$ in the EC-AFM cell is shown in Figure 1. The red curve, measured in a $\mathrm{N}_{2}$-protected atmosphere, shows the hydrogen region between 0.05 and 0.26 $\mathrm{V}$ with peaks at 0.12 and $0.21 \mathrm{~V}$ corresponding to the

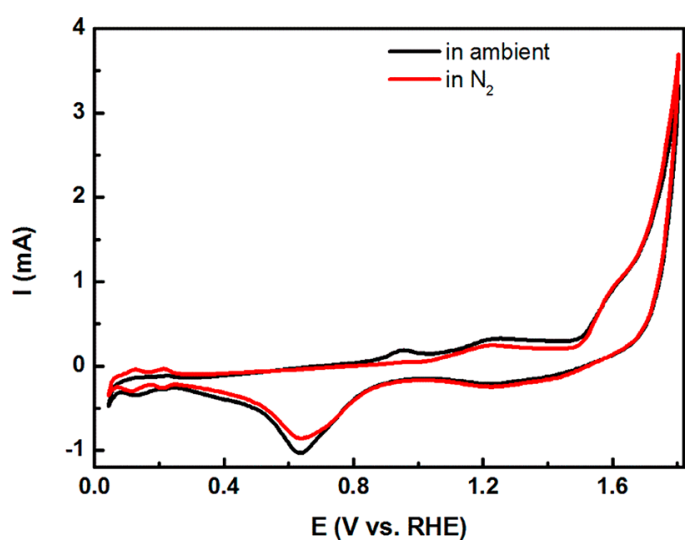

Figure 1. Cyclic voltammogram of $\mathrm{Pt}$ in $0.1 \mathrm{M} \mathrm{H}_{2} \mathrm{SO}_{4}$ in ambient atmosphere (black curve) and in $\mathrm{N}_{2}$-saturated electrolyte (red curve). Scan rate is $100 \mathrm{mV} / \mathrm{s}$.

adsorption of hydrogen at (110) and (100) steps, respectively. The oxidation of the Pt occurs when the potential increases to $1.0 \mathrm{~V}$, and the sharply increased current above $1.45 \mathrm{~V}$ indicates the evolution of oxygen. The negative-going sweep displays the reduction peak of the Pt oxide at $0.64 \mathrm{~V}$. There are two types of Pt oxide, $\alpha$-Pt oxide, which forms under moderate oxidation conditions, and $\beta$-Pt oxide, which forms under more severe oxidation conditions and which has a porous structure. ${ }^{16,17}$ The $\beta$-Pt oxide is expected to be reduced at a more negative potential than the reduction of the $\alpha$-Pt oxide, which normally takes place at around $0.75 \mathrm{~V}^{18}$ The peak at $0.64 \mathrm{~V}$ is attributed to the reduction of a mixture of $\alpha$ - and $\beta$-Pt oxides. As the AFM measurement is performed in an open EC cell without removal of the oxygen from the electrolyte, the black curve shows the $\mathrm{CV}$ recorded during the experiment under ambient atmosphere. As compared with the experiment under $\mathrm{N}_{2}$, the presence of oxygen leads to a small tilting of the curve (due to residual oxygen reduction) and the appearance of a small anodic peak at about $0.95 \mathrm{~V}$. We have performed also in situ EC-AFM measurements in which $\mathrm{N}_{2}$ was purged prior to the experiment, and the observations to be described below were the same.

AFM Imaging of Pt Surface after Potential Cycling. Figure 2 shows in situ EC-AFM topographical images of the platinum surface after potential cycling for 50 times in different potential ranges. The topography in Figure 2a shows terraces with sawtooth-shaped steps covering the whole imaging area, which we assign to a faceting induced during the flame annealing of the polycrystalline sample (its orientation is not defined in the experiment). There was no control over the orientation of the surface that was imaged, but the electrochemical phenomena are basically the same on all electrodes. Figure $2 \mathrm{a}$ and $\mathrm{b}$ show the images obtained after cycling 50 times between 0.05 and $1.0 \mathrm{~V}$ and between 0.05 and $1.5 \mathrm{~V}$, respectively. In all images, the potential of the Pt working electrode was held at $0.5 \mathrm{~V}$. Potential excursion to $1.5 \mathrm{~V}$ does not bring obvious changes to the surface as compared with the image obtained after cycling to $1.0 \mathrm{~V}$. Previous EC-STM investigations showed the formation of $\mathrm{Pt}$ islands on the $\mathrm{Pt}(111)$ surface after cycling five times between 0 and $1.5 \mathrm{~V}$ in $\mathrm{H}_{2} \mathrm{SO}_{4}$ electrolyte, ${ }^{8}$ but this roughening of the surface is not observed in our EC-AFM experiment, which we attribute to the polycrystalline nature of the sample and the lower resolution of the AFM as compared to STM. However, 

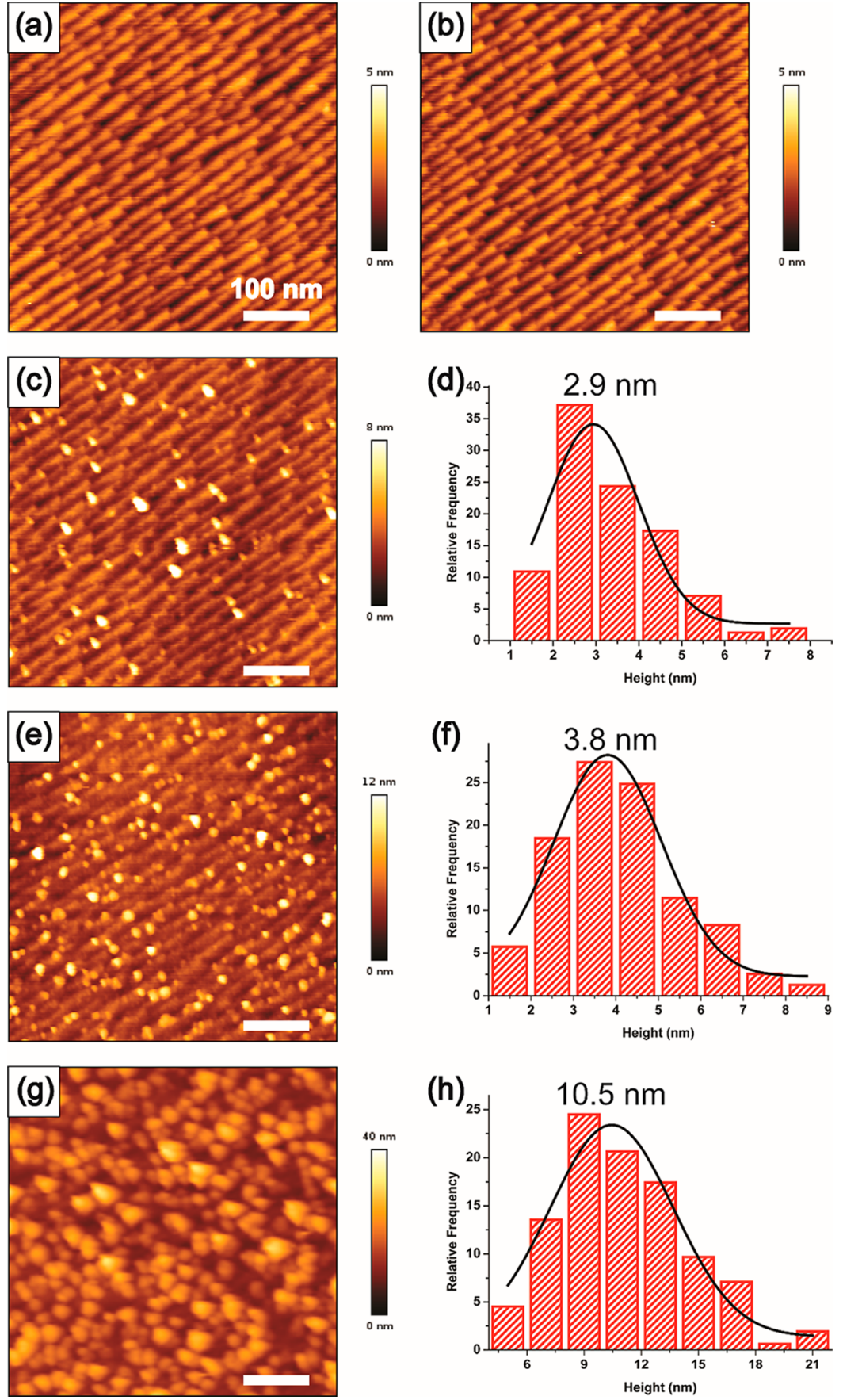

Figure 2. AFM height images of the Pt electrode surface after potential cycling between (a) $0.05-1.0 \mathrm{~V}$, (b) $0.05-1.5 \mathrm{~V}$, (c) $0.05-1.8 \mathrm{~V}$, (e) $0.05-$ $2.0 \mathrm{~V}$, and $(\mathrm{g}) 0.05-2.5 \mathrm{~V}$. The height distribution of the nanoparticles on the surface is shown in (d), (f), and (h). The scale bars are $100 \mathrm{~nm}$.

extending the upper potential limit to $1.8 \mathrm{~V}$ during the 50 oxidation-reduction cycles leads to a visible coarsening of the Pt surface through the appearance of nanoparticles on the surface (Figure 2c). The average size of the nanoparticles is estimated to be $2.9 \mathrm{~nm}$ by a statistical analysis at least 150 particles from the image. On increasing the upper potential to $2.0 \mathrm{~V}$, both the roughness of the surface and the number and the size of the nanoparticles increase (Figure 2e and f). The $t$ test result shows that the population mean of Figure $2 \mathrm{f}$ is significantly different from that of Figure $2 \mathrm{~d}$ at the 0.05 level of significance. Further sweeping to a potential of $2.5 \mathrm{~V}$ results in violent oxygen evolution and leads to the formation of nanoparticles with an average size of $10.5 \mathrm{~nm}$ covering the whole surface (Figure $2 \mathrm{~g}$ and $\mathrm{h}$ ). Increasing the upper potential during the cycling therefore increases the size of the nanoparticles, suggesting that the extensive oxidation of the electrode promotes the formation of the nanoparticles.

To investigate the minimum upper potential needed for the observation of the nanoparticles, experiments were carried out by increasing the upper potential in $0.1 \mathrm{~V}$ steps while keeping the lower potential at $0.05 \mathrm{~V}$. Figure 3 shows that after potential cycling between 0.05 and $1.7 \mathrm{~V}$ (Figure 3a) no particles are observed, but the surface shows the appearance of nanoparticles after cycling up to $1.8 \mathrm{~V}$ (Figure $3 \mathrm{~b}$ ). Therefore, the minimum upper potential for the formation of nanoparticles under the current experimental conditions is between 

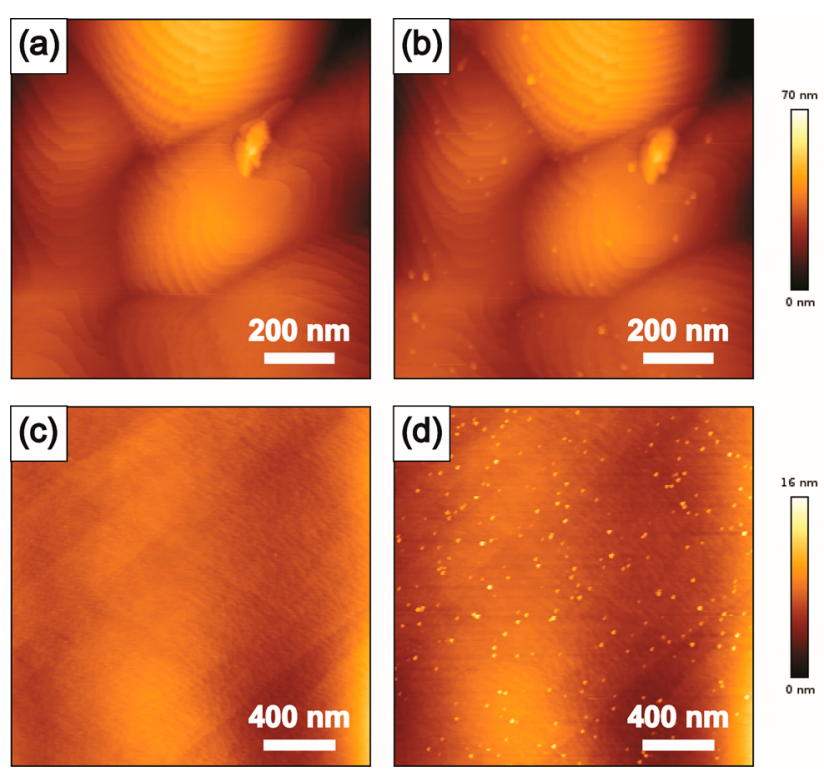

Figure 3. AFM height images of the Pt electrode surface after potential cycling between (a) $0.05-1.7 \mathrm{~V}$, (b) $0.05-1.8 \mathrm{~V}$, (c) 0.9$1.8 \mathrm{~V}$, and $(\mathrm{d}) 0.8-1.8 \mathrm{~V}$. Note that the scale is not the same in these images.

1.7 and $1.8 \mathrm{~V}$. Similarly, there is a maximum lower potential to observe nanoparticles, which is found to be $0.8 \mathrm{~V}$, as illustrated in Figure $3 \mathrm{c}$ and $\mathrm{d}$. Accordingly, to observe the formation of the nanoparticles, the potential cycling range should be set beyond the window of $0.8-1.8 \mathrm{~V}$.

In Situ AFM Observation of the Nanoparticles during Potential Cycling. To further clarify the formation of the nanoparticles, in situ EC-AFM imaging of the Pt surface was performed simultaneously with the potential cycling. In Figure $4 \mathrm{a}$, the tip is scanned from top to bottom while the potential is cycled. Two different strips or areas can be observed in the image, bordered by the white dashed lines. Nanoparticles are observed in one strip, but no nanoparticles are observed in the neighboring strip. Scanning positively, the nanoparticles disappear above $1.35 \mathrm{~V}$ and reappear again in the subsequent negative-going scan below $0.95 \mathrm{~V}$. The observation of nanoparticles is indicated by the red line in the corresponding cyclic voltammogram in Figure $4 \mathrm{~b}$. No nanoparticles are observed on the surface in the black part of the CV. In order to rule out the possibility that the nanoparticles are detached from the surface due to the interference of the AFM tip, an experiment was performed by keeping the potential at $1.4 \mathrm{~V}$ for
5 min, on a surface that contained nanoparticles. The AFM image obtained after the treatment showed no nanoparticles on the surface (Figure S1). Moreover, it is found that potential cycling between 0.05 and $1.2 \mathrm{~V}$ reduces the number of nanoparticles and their size shrinks. Cycling between 0.05 and $1.4 \mathrm{~V}$ removes the nanoparticles on the $\mathrm{Pt}$ surface altogether, but they reappear after cycling between 0.05 and $1.8 \mathrm{~V}$, confirming the above-mentioned minimum upper potential for the formation of nanoparticles (see Figure S2). On the basis of these observations we conclude that the nanoparticles are indeed Pt nanoparticles and that the onset of the nanoparticle formation occurs at $0.95 \mathrm{~V}$, in agreement with the onset of $\mathrm{Pt}$ ion production due to the reduction of the $\mathrm{Pt}$ oxide as studied by online ICP-MS. ${ }^{7,19}$ The oxidation of Pt nanoparticles begins at about $1.2 \mathrm{~V}$, leading to their decrease in number and size during potential cycling between 0.05 and $1.2 \mathrm{~V}$, and they completely disappear when cycling up to $1.4 \mathrm{~V}$. Moreover, there is no significant effect of the scanning tip in the removal of the nanoparticles. These results show that the $\mathrm{Pt}$ nanoparticles are in full electronic contact with the working electrode, and hence their oxidation state varies with the potential in the same way as the rest of the platinum surface. We note that recent work has shown that there might be $\mathrm{OH}$ existing on the steps in a platinum (nanoparticle) surface at 0.5 $\mathrm{V}^{20,21}$

The upper potential of our experiment is within the potential window in which oxygen is generated. The presence of oxygen in the electrolyte may enhance $\mathrm{Pt}$ dissolution and $\mathrm{Pt}$ ion formation. ${ }^{22-24}$ Reduction of oxygen in the negative-going sweep can produce hydroxyl radicals $\left(\mathrm{OH}^{\bullet}\right)$ according to reaction $1 .{ }^{25}$ It has been suggested by Percival et al. that highly oxidizing $\mathrm{OH}^{\bullet}$ is responsible for the Pt dissolution during the oxygen reduction reaction. In our experiment, the formation of Pt nanoparticles starts at $0.95 \mathrm{~V}$ vs $\mathrm{RHE}$ at $\mathrm{pH} \mathrm{1,} \mathrm{a} \mathrm{potential}$ higher than the hydroxyl formation potential. The investigation from Noël et al. reveals the important role of hydroxyl radical for platinum dissolution; however, they found no appreciable dissolution of $\mathrm{Pt}$ even after a long sequence of potential pulses in acidic solutions $(\mathrm{pH} \leq 2.5){ }^{26}$

$$
\begin{gathered}
\mathrm{O}_{2}+2 \mathrm{H}^{+}+3 \mathrm{e}^{-} \rightarrow \mathrm{OH}^{\bullet}+\mathrm{OH}^{-} \\
E^{0}=0.39 \mathrm{~V} \text { vs NHE at } \mathrm{pH} 7
\end{gathered}
$$

Our interpretation of the potential limits for nanoparticle formation is that while the existence of thick Pt oxide is the precondition for the formation of $\mathrm{Pt}$ nanoparticles, the nanoparticles are actually formed during the reductive sweep.
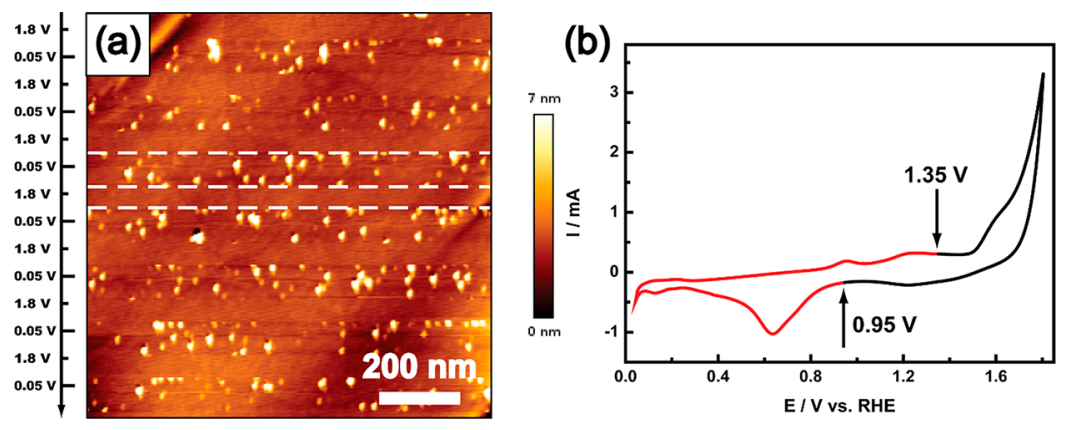

Figure 4. (a) AFM height image of the Pt surface during potential cycling between 0.05 and $1.8 \mathrm{~V}$ illustrating the potential-dependent formation of the nanoparticles. The $y$ axis gives the potential during the potentiodynamic cycling. (b) Corresponding CV of the Pt electrode. The red part of the $\mathrm{CV}$ shows the potentials for which the AFM image shows nanoparticles on the surface, while in the black part no nanoparticles are observed. 

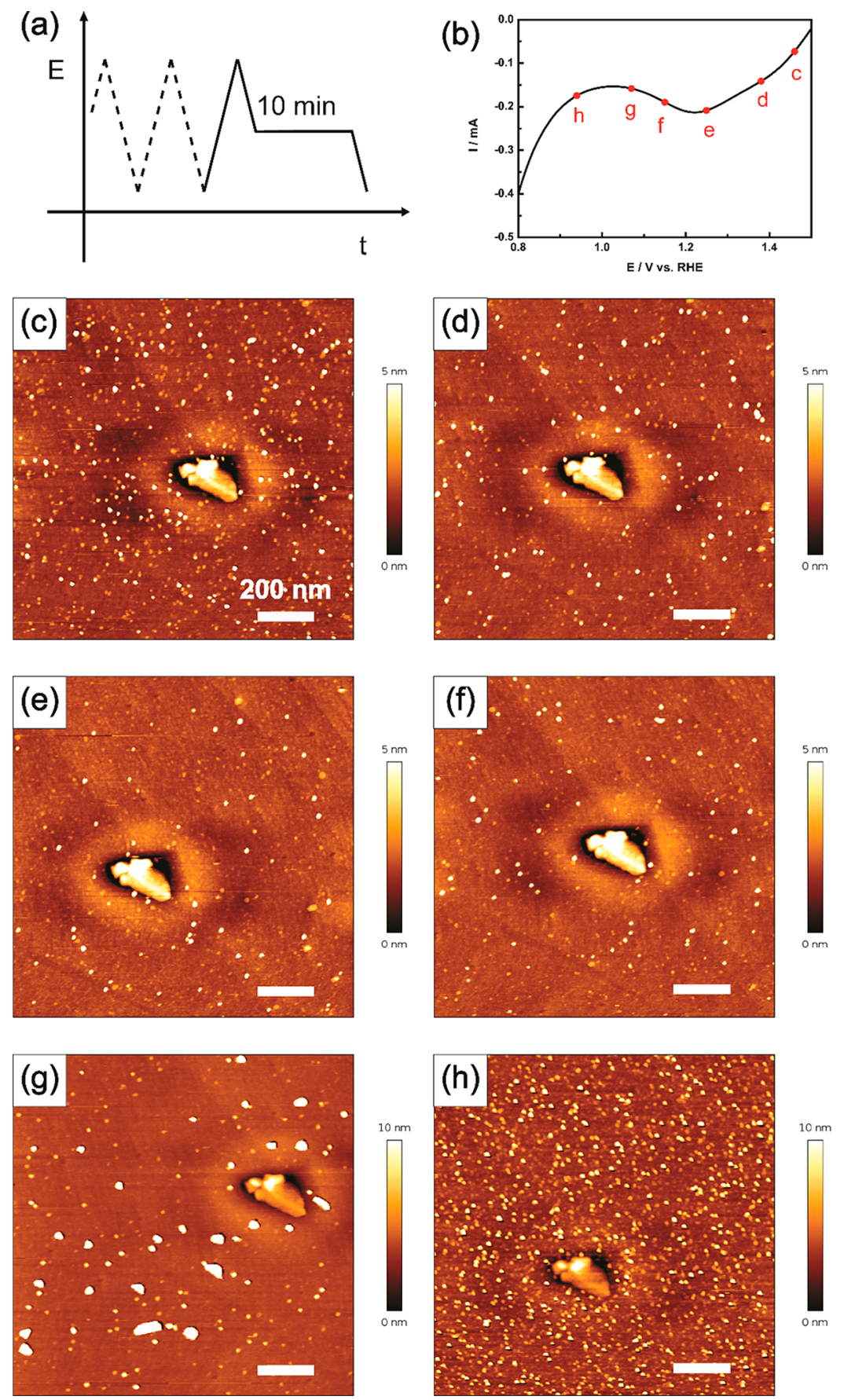

Figure 5. (a) Potential protocol used to control the formation of Pt nanoparticles on the surface. (b) The six potential values chosen as the stopping potential, which are 1.46, 1.38, 1.25, 1.15, 1.07, and $0.94 \mathrm{~V}$, respectively. $(\mathrm{c}-\mathrm{h})$ AFM height images of the Pt surface obtained after applying the protocol corresponding to points $\mathrm{c}-\mathrm{h}$ in (b). The scale bars are $200 \mathrm{~nm}$.

Figure 5 shows results of experiments in which during the negative scan the potential is held at a certain value ("the stopping potential") for $10 \mathrm{~min}$ before continuing to $0.05 \mathrm{~V}$ (Figure 5a); the six potentials are labeled in the CV curve (Figure $5 b$ ). The six surface images shown in Figure $5 c-h$ are obtained (at $0.5 \mathrm{~V}$ ) after following this protocol for the different stopping potentials. An area with what appears to be a triangular defect structure was imaged for comparison; however, the triangles in the AFM images are not in the exact same position due to thermal drift. It is observed that as the stopping potential decreases from Figure $5 \mathrm{c}$ to e, the number of $\mathrm{Pt}$ nanoparticles decreases as well. Figure 5e and $\mathrm{f}$ have a similar density of nanoparticles. As seen from the enlarged $\mathrm{CV}$ curve in Figure $5 \mathrm{~b}$, stopping potentials $\mathrm{c}$ to $\mathrm{f}$ are within the potential window where the $\mathrm{Pt}$ oxide is reduced, creating Pt ions in solution at the same time. ${ }^{7,19,27}$ Once the Pt ions are formed next to the Pt electrode, diffusion will carry them away from the surface if further reduction does not take place, which explains why fewer nanoparticles are observed on the surface in Figure $5 \mathrm{c}$ to e. Interestingly, when the potential is held at $1.07 \mathrm{~V}$ for $10 \mathrm{~min}$, large nanoparticles with a height of up to $30 \mathrm{~nm}$ appear on the surface, as shown in Figure $5 \mathrm{~g}$. By contrast, when the stopping potential is $0.94 \mathrm{~V}$ for $10 \mathrm{~min}$, a very large amount of small nanoparticles deposit on the surface 
(Figure $5 \mathrm{~h}$ ). As stated before, $0.95 \mathrm{~V}$ is the potential at which nanoparticles begin to appear during the cycling, which means that at around $0.95 \mathrm{~V}$ the nucleation of $\mathrm{Pt}$ nanoparticles starts. Holding the potential at $0.94 \mathrm{~V}$ for $10 \mathrm{~min}$ provokes the instantaneous nucleation of $\mathrm{Pt}$, which leads to the formation of a substantial number of small Pt nanoparticles. On the other hand, holding the potential at $1.07 \mathrm{~V}$, there is a very small number of nucleation sites, and as a result, the nanoparticles grow bigger than in the case of a larger number of nucleation sites. These observations confirm a nucleation and growth mechanism of nanoparticle formation in which the number of nucleation sites depends on the extent to which the Pt oxide has been reduced, and the growth of the Pt nanoparticles is influenced by the diffusion of dissolved $\mathrm{Pt}$ ions away from the electrode surface.

Based on our observations, a model for the formation of nanoparticles is proposed, as illustrated in Scheme 1. First, the

Scheme 1. Proposed Mechanism for Pt Nanoparticle

Formation on the Surface in $\mathrm{H}_{2} \mathrm{SO}_{4}$ Electrolyte

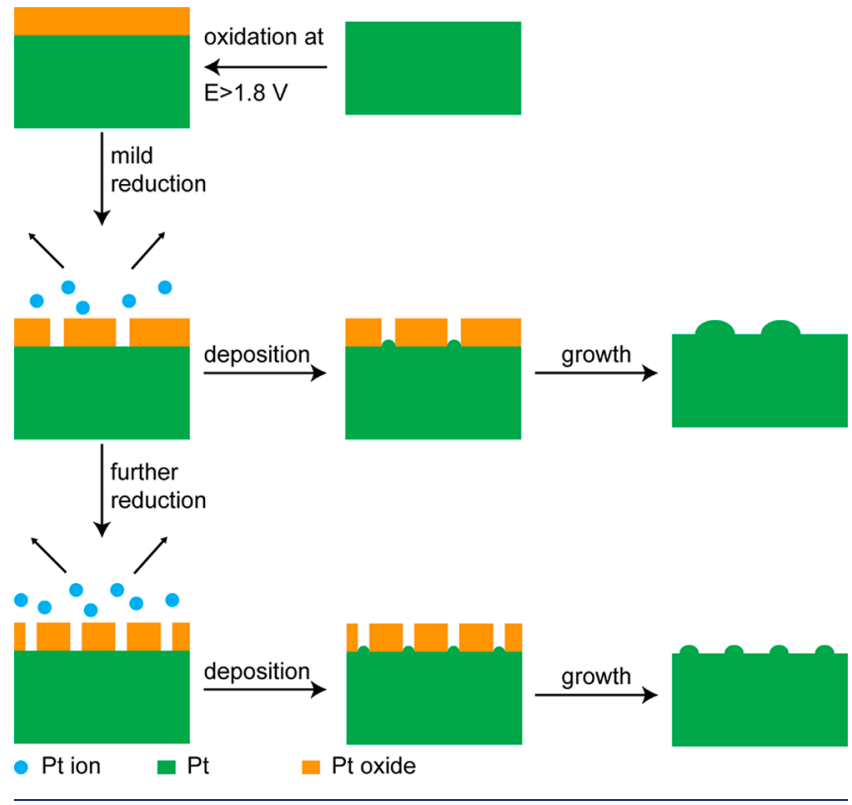

Pt surface is oxidized at a potential higher than $1.8 \mathrm{~V}$, which forms a thick Pt oxide layer. In the negative scan, reduction of the oxide leads to the formation of $\mathrm{Pt}$ ions close to the surface, which deposit as $\mathrm{Pt}$ nanoparticles in the further reduction process. The minimum potential of $1.8 \mathrm{~V}$ is assumed to be related to a minimum oxide thickness that generates a minimum concentration of dissolved $\mathrm{Pt}$ near the electrode during the reduction of the oxide. The formation of the nanoparticles strongly depends on the extent to which Pt oxide is reduced. If only a small amount of oxide is reduced, this leads to limited nucleation sites for Pt deposition. Therefore, fewer but larger nanoparticles are found on the surface in this case. When the Pt oxide is further reduced, more nucleation sites are generated before the massive deposition of $\mathrm{Pt}$, leading to many small nanoparticles. In the following potential cycle, the Pt surface is oxidized at high potential, which forms the thick oxide layer again. The continuous oxidation and reduction creates the oxide layer and $\mathrm{Pt}$ ions, which leads to the periodical behavior of the disappearance and reappearance of nanoparticles as observed in the in situ AFM image shown in Figure 4.

\section{CONCLUSION}

In summary, we have used in situ EC-AFM to investigate the formation of $\mathrm{Pt}$ nanoparticles on the surface of a polycrystalline flame-annealed $\mathrm{Pt}$ electrode in $0.1 \mathrm{M} \mathrm{H}_{2} \mathrm{SO}_{4}$, during various potential excursions between 0.05 and $2.5 \mathrm{~V}$. The size and the number of the nanoparticles increase with the upper potential limit. A minimal upper potential of $1.8 \mathrm{~V}$ was observed, which we ascribe to the existence of a thick oxide layer as a precondition for the formation of the Pt nanoparticles in the subsequent negative-going scan. Reduction of this oxide layer generates dissolved $\mathrm{Pt}$ ions, which will start to electrodeposit by nucleation and growth on the surface at about $0.95 \mathrm{~V}$, this potential being related to the reduction of the surface oxide. A thick enough oxide and its subsequent reduction are therefore vital to the $\mathrm{Pt}$ nanoparticle formation. We note that this mechanism of surface roughening is different from the mechanism studied recently by in situ GISAXS and ECSTM, which studied platinum surface roughening in a more limited potential window in which the redeposition of dissolved Pt (presumably) plays a minor role. The generation of nanoparticles is observed when cycling a Pt electrode in acidic electrolyte, which is the most common electrolyte used in Pt electrochemistry. It will be of great interest to study the phenomenon in other electrolytes, including buffer solutions and alkaline media. This study also demonstrates the important role that in situ EC-AFM can play in investigating (dynamic) morphological changes of Pt under electrochemical control, which will ultimately help to understand and improve the performance of the Pt-based devices for electrochemical energy conversion and electroanalysis. Due to the ability to make observations of surface morphology in real space and time, we hope that our study will inspire more in situ EC-AFM investigations of electrochemical surfaces.

\section{ASSOCIATED CONTENT}

\section{Supporting Information}

The Supporting Information is available free of charge on the ACS Publications website at DOI: 10.1021/jacs.8b07452.

AFM images of $\mathrm{Pt}$ nanoparticles on the $\mathrm{Pt}$ electrode surface after potential holding and potential cycling (PDF)

\section{AUTHOR INFORMATION}

\section{Corresponding Author}

*m.koper@chem.leidenuniv.nl

ORCID

Marc T. M. Koper: 0000-0001-6777-4594

Notes

The authors declare no competing financial interest.

\section{ACKNOWLEDGMENTS}

This work received partial support from Hitachi, Ltd. and Hitachi High-Technologies Corporation.

\section{REFERENCES}

(1) Borup, R.; Meyers, J.; Pivovar, B.; Kim, Y. S.; Mukundan, R.; Garland, N.; Myers, D.; Wilson, M.; Garzon, F.; Wood, D.; Zelenay, P.; More, K.; Stroh, K.; Zawodzinski, T.; Boncella, J.; McGrath, J. E.; Inaba, M.; Miyatake, K.; Hori, M.; Ota, K.; Ogumi, Z.; Miyata, S.; Nishikata, A.; Siroma, Z.; Uchimoto, Y.; Yasuda, K.; Kimijima, K.-i.; Iwashita, N. Chem. Rev. 2007, 107, 3904-3951. 
(2) Wang, Y.-J.; Wilkinson, D. P.; Zhang, J. Chem. Rev. 2011, 111, $7625-7651$.

(3) Yu, X.; Ye, S. J. Power Sources 2007, 172, 145-154.

(4) Debe, M. K. Nature 2012, 486, 43-51.

(5) Huang, Y. F.; Kooyman, P. J.; Koper, M. T. Nat. Commun. 2016, $7,12440$.

(6) Komanicky, V.; Chang, K. C.; Menzel, A.; Markovic, N. M.; You, H.; Wang, X.; Myers, D. J. Electrochem. Soc. 2006, 153, B446-B451.

(7) Topalov, A. A.; Katsounaros, I.; Auinger, M.; Cherevko, S.; Meier, J. C.; Klemm, S. O.; Mayrhofer, K. J. J. Angew. Chem., Int. Ed. 2012, 51, 12613-12615.

(8) Breuer, N.; Funtikov, A. M.; Stimming, U.; Vogel, R. Surf. Sci. 1995, 335, 145-154.

(9) Furuya, N.; Shibata, M. J. Electroanal. Chem. 1999, 467, 85-91.

(10) Furuya, N.; Ichinose, M.; Shibata, M. J. Electroanal. Chem. 1999, 460, 251-253.

(11) Ruge, M.; Drnec, J.; Rahn, B.; Reikowski, F.; Harrington, D. A.; Carlà, F.; Felici, R.; Stettner, J.; Magnussen, O. M. J. Am. Chem. Soc. 2017, 139, 4532-4539.

(12) Wakisaka, M.; Asizawa, S.; Uchida, H.; Watanabe, M. Phys. Chem. Chem. Phys. 2010, 12, 4184-4190.

(13) Jacobse, L.; Huang, Y.-F.; Koper, M. T. M.; Rost, M. J. Nat. Mater. 2018, 17, 277-282.

(14) Khalakhan, I.; Choukourov, A.; Vorokhta, M.; Kúš, P.; Matolínová, I.; Matolín, V. Ultramicroscopy 2018, 187, 64-70.

(15) Onochi, Y.; Nakamura, M.; Hoshi, N. J. Phys. Chem. C 2012, $116,15134-15140$.

(16) Tremiliosi-Filho, G.; Jerkiewicz, G.; Conway, B. E. Langmuir 1992, 8, 658-667.

(17) Shibata, S. J. Electroanal. Chem. Interfacial Electrochem. 1978, 89, $37-58$.

(18) Mitsushima, S.; Kawahara, S.; Ota, K.-i.; Kamiya, N. J. Electrochem. Soc. 2007, 154, B153-B158.

(19) Cherevko, S.; Topalov, A. A.; Zeradjanin, A. R.; Keeley, G. P.; Mayrhofer, K. J. J. Electrocatalysis 2014, 5, 235-240.

(20) Huang, Y.-F.; Koper, M. T. M. J. Phys. Chem. Lett. 2017, 8, $1152-1156$

(21) Chen, X.; McCrum, I. T.; Schwarz, K. A.; Janik, M. J.; Koper, M. T. M. Angew. Chem., Int. Ed. 2017, 56, 15025-15029.

(22) Matsumoto, M.; Miyazaki, T.; Imai, H. J. Phys. Chem. C 2011, $115,11163-11169$.

(23) Bae, J. H.; Brocenschi, R. F.; Kisslinger, K.; Xin, H. L.; Mirkin, M. V. Anal. Chem. 2017, 89, 12618-12621.

(24) Percival, S. J.; Dick, J. E.; Bard, A. J. Anal. Chem. 2017, 89, 3087-3092.

(25) Noël, J.-M.; Latus, A.; Lagrost, C.; Volanschi, E.; Hapiot, P. J. Am. Chem. Soc. 2012, 134, 2835-2841.

(26) Noël, J.-M.; Yu, Y.; Mirkin, M. V. Langmuir 2013, 29, 13461350.

(27) Cherevko, S.; Zeradjanin, A. R.; Topalov, A. A.; Kulyk, N.; Katsounaros, I.; Mayrhofer, K. J. J. ChemCatChem 2014, 6, 22192223. 